\title{
Atomic and Electronic Edge Structures of Monolayer Ceria on $\operatorname{Pt}(111)$
}

Zhichao Huang, ${ }^{1,2}$ Cheng Han, ${ }^{* 1}$ Yi-Yang Sun, ${ }^{* 4}$ Kai Wu, ${ }^{* 3}$ and Wei Chen ${ }^{* 2,5,6}$

${ }^{1}$ SZU-NUS Collaborative Innovation Center for Optoelectronic Science \& Technology, International Collaborative Laboratory of 2D Materials for Optoelectronics Science and Technology of Ministry of Education, Institute of Microscale Optoelectronics, Shenzhen University, Shenzhen 518060, China.

${ }^{2}$ Department of Physics, National University of Singapore, 2 Science Drive 3, Singapore 117542, Singapore.

${ }^{3}$ BNLMS, College of Chemistry and Molecular Engineering, Peking University, Beijing 100871, China.

${ }^{4}$ State Key Laboratory of High Performance Ceramics and Superfine Microstructure Shanghai Institute of Ceramics, Chinese Academy of Sciences, Shanghai 201899, China.

${ }^{5}$ Department of Chemistry, National University of Singapore, 3 Science Drive 3, Singapore 117543, Singapore.

${ }^{6}$ Joint School of National University of Singapore and Tianjin University, International Campus of Tianjin University, Binhai New City, Fuzhou, 350207, China

E-mails:phycw@nus.edu.sg,yysun@mail.sic.ac.cn, hancheng@szu.edu.cn and kaiwu@pku.edu.cn 
(a)

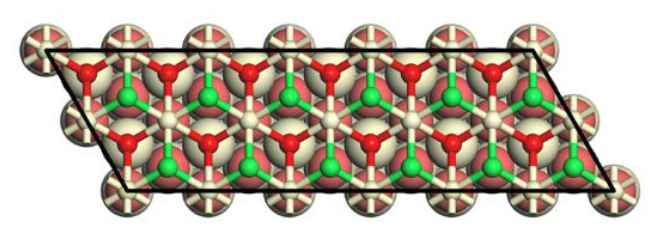

(c)

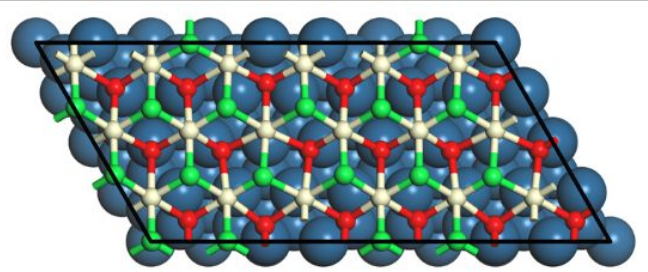

(b)

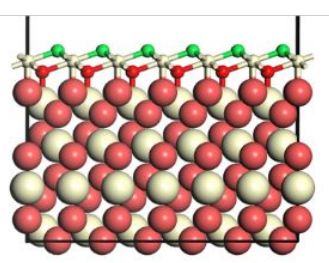

(d)

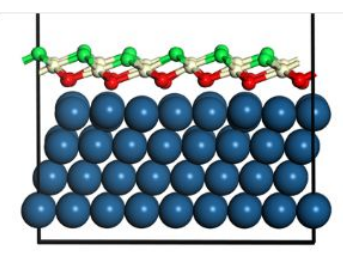

internal $\mathrm{O}^{2-}$

$\mathrm{O}_{i}^{2-}$

$\mathrm{O}_{\mathrm{s}}^{2-}$

$\mathrm{Ce}^{4+}$

- Pt

Figure S1. (a) Top view and (b) side view of optimized $\mathrm{CeO}_{2}(111)$ slab models. (c) Top view and (d) side view of optimized $\mathrm{CeO}_{2} / \mathrm{Pt}(111)$ slab models. The light red balls, red balls, green balls, white balls and blue balls correspondingly represent the internal oxygen ions, $\mathrm{O}_{\mathrm{i}}^{2-}$ ions, $\mathrm{O}_{\mathrm{s}}^{2-}$ ions, $\mathrm{Ce}^{4+}$ ions and $\mathrm{Pt}$ atoms. The outmost $\mathrm{O}-\mathrm{Ce}-\mathrm{O}$ layer is presented in a ball-and-stick style.
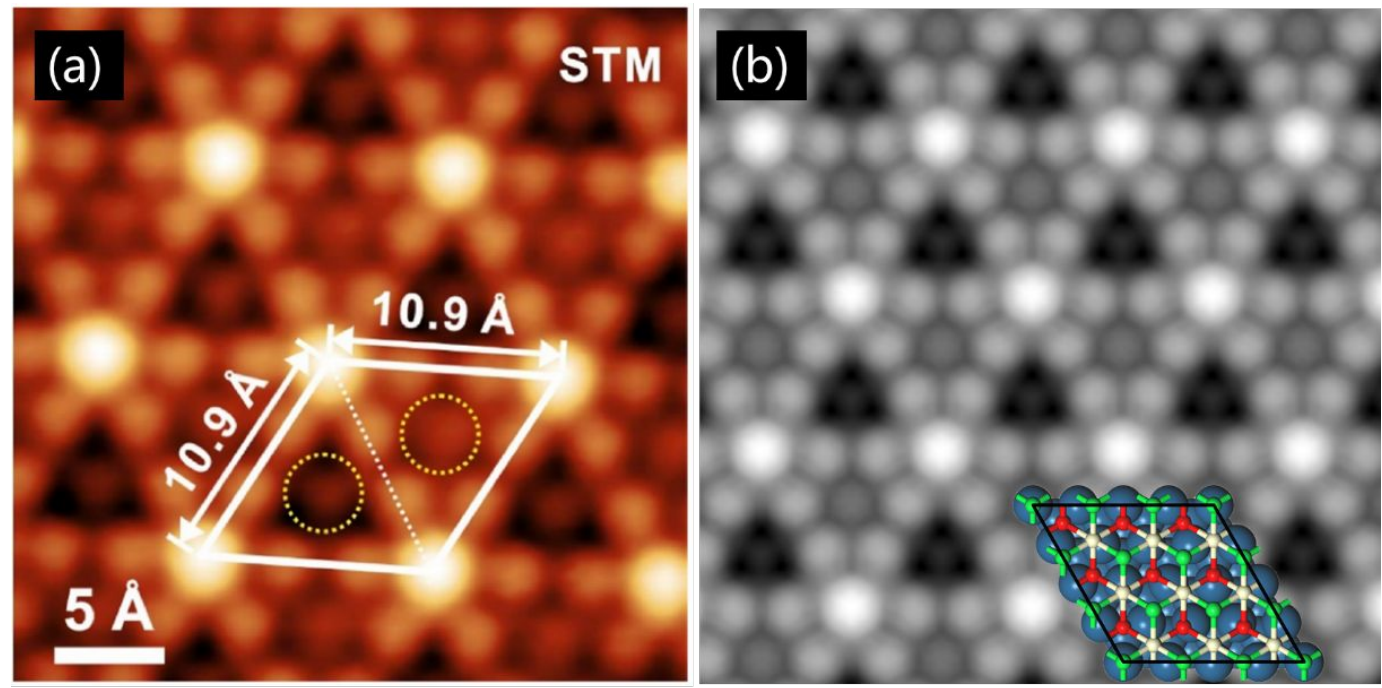

Figure S2. Experimental and simulated STM image of monolayered $\mathrm{CeO}_{2} / \mathrm{Pt}(111)$. All bright spots are surface oxygen atoms. (a) High-resolution experimental image. Reprinted from ref. 1, with permission from the American Chemical Society, copyright 2020. (b) Simulation image in this article, which is highly consistent with the experimental results. 


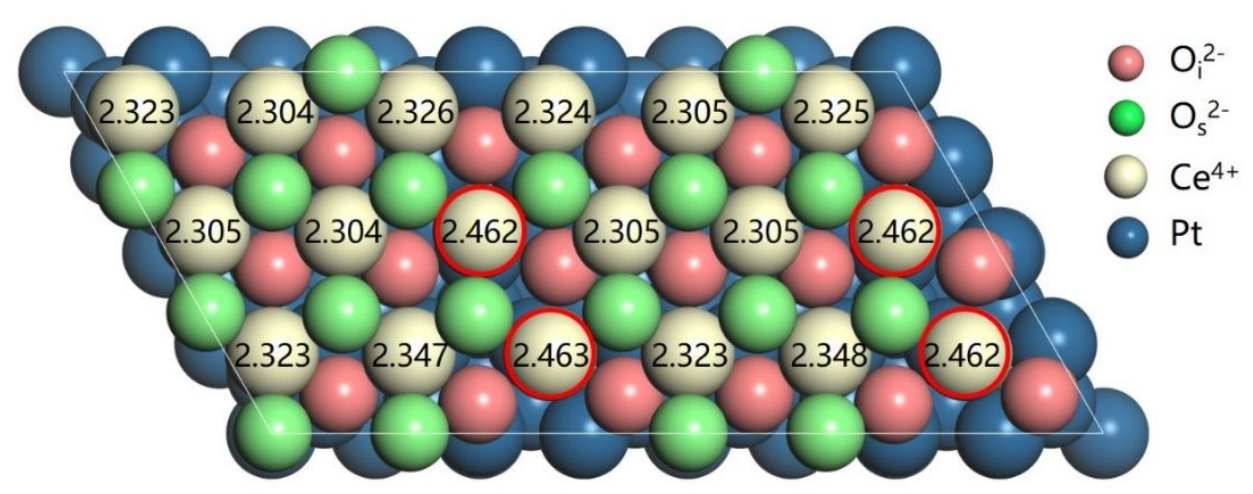

Figure S3. The average distance between each $\mathrm{Ce}^{4+}$ ion and its neighboring $\mathrm{O}^{2-}$ ions in the $\mathrm{CeO}_{2} / \mathrm{Pt}(111)$ model. The red balls, green balls, white balls, and blue balls represent $\mathrm{O}_{\mathrm{i}}{ }^{2-}$ ions, $\mathrm{O}_{\mathrm{s}}{ }^{2-}$ ions, $\mathrm{Ce}^{4+}$ ions, and $\mathrm{Pt}$ atoms separately. Four Ce ions with comparatively large average distances are circled in red.

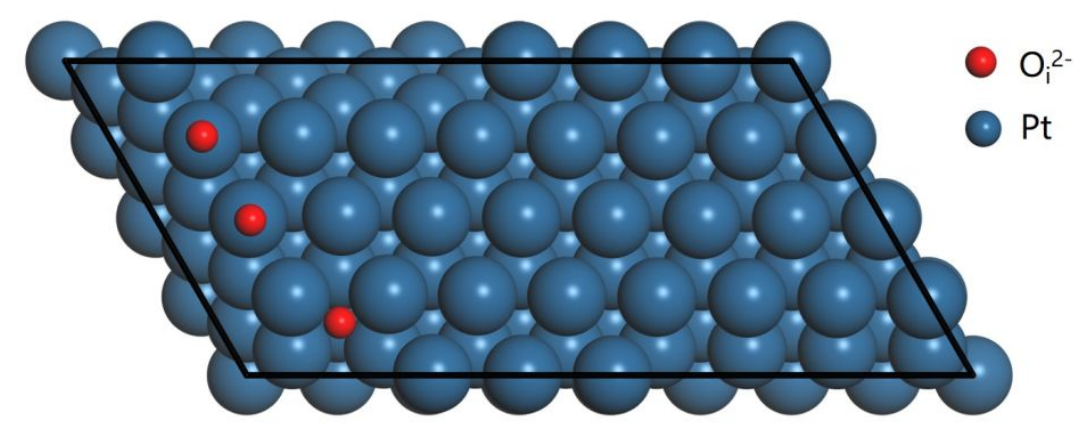

Figure S4. O-row/Pt(111) model, the blue ball and the red ball represent $\mathrm{Pt}$ atoms and $\mathrm{O}_{\mathrm{i}}^{2-}$ ions resectively. 
(a)

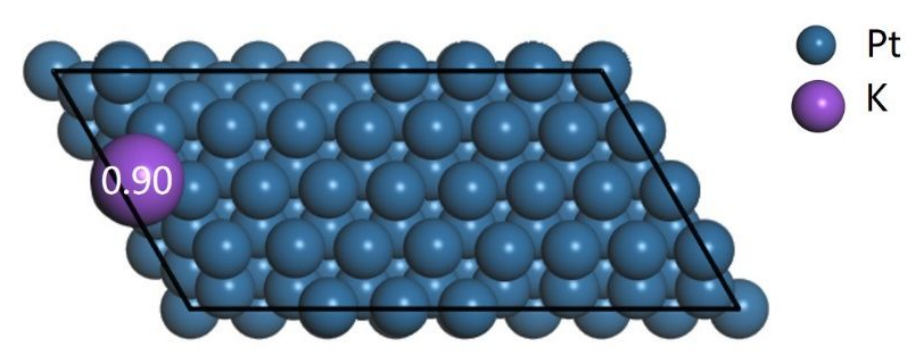

(b)

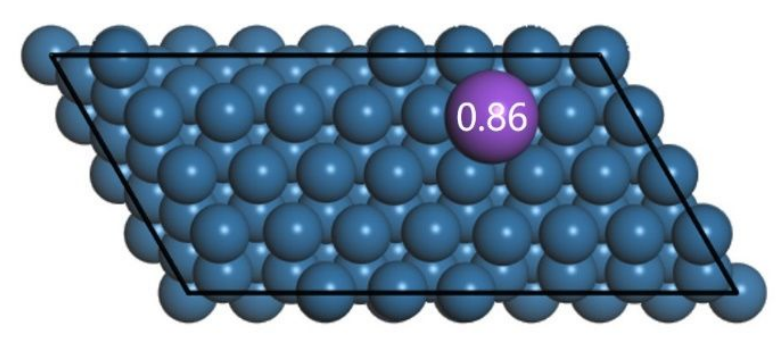

Figure S5. K/Pt(111) model, the position of $\mathrm{K}$ ion is the same as at (a) $\mathrm{CeO}_{2} / \mathrm{Pt}(111)$ $\mathrm{KL} 1 \mathrm{R} 0$ and (b) $\mathrm{CeO}_{2} / \mathrm{Pt}(111)-\mathrm{L} 1 \mathrm{R} 0 \mathrm{~K}$ models in Figure 6 . The blue ball and the purple ball represent $\mathrm{Pt}$ atoms and $\mathrm{K}$ ions respectively. The number marked on the $\mathrm{K}$ ion is its Bader charge.

\section{REFERENCES}

(1) Chen, H.; Huang, Z.; Rong, W.; Zhou, J.; Xu, Z.; Li, W.; Lin, Y.; Zhou, X.; Wu, K. Atomically Resolved Structure of Monolayer Ceria Island on Pt(111). J. Phys. Chem. C 2020, 124, 28531-28538. 